\title{
COMPORTAMENTO DE DOIS CULTIVARES DE SOJA EM FUNÇÃO DO MANGANES DO SOLO ${ }^{(1)}$
}

Manoel Albino Coelho de Miranda $\left({ }^{2}\right)$, Hipólito Assunção Antonio MascareNhas $\left({ }^{2}\right)$, Eduardo Antonio Bulisani $\left({ }^{2}\right)$, Seçāo de Leguminosas, José Maria Aires da Silva Valadares $\left({ }^{2}\right)$, Seção de Pedologia, e Rúter Hiroce, Seçāo de Quimica Analítica, Instituto Agronômico

\section{RESUMO}

Foi estudado, em casa de vegetação, o comportamento dos cultivares de soja Biloxi e Forrest, em amostras subsuperficiais de Latossolo Roxo distrófico dos municípios paulistas de Campinas, Guaíra, Guatapará e Cândido Mota, contendo respectivamente $2,9,6,4,11,3$ e $12,9 \mathrm{ppm}$ de $\mathrm{Mn}$ solúvel em ácido dietilenotriaminopentacético (DTPA). A expressão da toxicidade de Mn foi avaliada 27 dias após o plantio, através de notas atribuídas aos sintomas visuais; produção de matéria seca das raízes e parte aérea; e da análise química da parte aérea. O cultivar Biloxi não apresentou sintomas de toxicidade até ao nível de 6,4 ppm de $\mathrm{Mn}$ no solo (Guaíra), enquanto o 'Forrest' já os apresentava nesse nível. A medida que se tornavam mais elevados os teores de $\mathrm{Mn}$ no solo, mais agudos se apresentavam os sintomas de toxicidade e menor a produção de matéria seca, sendo que o 'Forrest' mostrou muito maior sensibilidade, o que foi confirmado pelo aumento do teor de $\mathrm{Mn}$ na parte aérea. Sendo eficiente na absorçáo do $\mathrm{Mn}$ do solo e na manifestaçăo dos sintomas de toxicidade a níveis baixos deste elemento, o 'Forrest' é o cultivar adequado para utilizar como indicador de niveis tóxicos de $\mathrm{Mn}$. A concordância entre as notas atribuídas aos sintomas visuais, à produçăo de matéria seca e ao índice (teor de $\mathrm{Mn}$ /peso seco), demonstra que as notas podem ser critério apropriado para realizar o "screening" de material genético num programa de melhoramento, para tolerância a Mn tóxico.

\section{INTRODUÇÃO}

E sabido que a toxicidade de manganês em solos ácidos, além de prejudicar o desenvolvimento da so- ja, tem efeito prejudicial e específico sobre a nodulação (9). Solos com teores tóxicos deste elemento têm sido relatados por COUTINHO et alii (6) no Rio Grande do Sul, AL-

(1) Trabalho apresentado no Congresso Brasileiro de Ciência do Solo em Salvador (BA) de 30 de agosto a 5 de setembro de 1981. Recebido para publicação a 5 de agosto de 1981 .

(2) Com bolsa de suplementação do CNPq. 
MEIDA e SFREDO (1) no Paraná, e MASCARENHAS et alii (15) em São Paulo. VALADARES \& CAMARGO $\left({ }^{3}\right)$ mostraram que, em solos derivados de rochas básicas, os teores de Mn extraído com DTPA de amostragem do horizonte $\mathbf{B}$, variaram de 4 a 24 ppm.

Trabalhos têm indicado que há variação de comportamento entre as espécies de plantas aos níveis tóxicos de Mn $(2,14,18)$. Evidências de diferenças entre cultivares à elevada concentração de $\mathrm{Mn}$ existem em trigo (7), algodão (8) e alfafa (21). Em soja, tanto HEENAN \& CARTER (10) quanto CARTER et alii (5) demonstraram que o 'Bragg' é muito suscetível e, 'Lee', bastante tolerante à toxicidade de manganês. BROWN \& JONES (4) confirmam essas observações, incluindo o 'Forrest' também como bastante suscetível ao Mn. Em trabalho mais recente, OHKI et alii (19) incluíram também esse cultivar entre aqueles para estudar o efeito da deficiência de $\mathrm{Mn}$ em solução nutritiva. Foi observado que, exceto o 'Forrest', os demais cultivares testados mostraram-se sensíveis à deficiência de $\mathrm{Mn}$ em níveis extremamente baixos.

$\mathrm{Na}$ maioria dos experimentos de competição de cultivares de soja conduzidos em Latossolo Roxo, no Estado de São Paulo, pela Seção de Leguminosas do Instituto Agronômico, o 'Forrest' foi o único que mostrou sintomas de necroses de nervura e enrugamento de folhas. Por outro lado, estudos feitos em solução nutritiva (16) com os cultivares Biloxi, Davis e Santa-Rosa mostra- ram que o primeiro foi o mais tolerante ao $\mathrm{Mn}$.

O objetivo deste trabalho foi verificar o comportamento dos cultivares Biloxi e Forrest em solos com diferentes teores de $\mathrm{Mn}$ obtidos de quatro localidades paulistas.

\section{MATERIAL E METODO}

$O$ experimento foi instalado em casa de vegetação, utilizando-se amostras de Latossolo Roxo distrófico provenientes de quatro municípios paulistas, em um esquema estatístico de parcelas subdivididas casualizadas, com seis repetiçōes. Foi aplicado um grama de sal $\mathrm{KH}_{2} \mathrm{PO}_{4}$ por vaso com $4 \mathrm{~kg}$ de terra. As amostras do solo foram coletadas de 20 a $40 \mathrm{~cm}$ de profundidade, misturando-se bem o sal com o solo. Em seguida, foram tiradas amostras de solo para efetuar a análise química, segundo os métodos de RAIJ \& ZULLO (22). Os teores de Mn foram obtidos pelo método preconizado por LINDSAY \& NORWEI.L (13), usando como solução extratcra o DTPATEA a pH 7,3, e a determinação por espectrofotometria de absorção atômica, sendo os resultados apresentados no quadro 1. Pelo fato de a temperatura influir na manifestação dos sintomas de toxicidade de $\mathrm{Mn}$ (11), o ensaio foi conduzido nos meses mais frios, quando a temperatura do ar registrada em termógrafo variou de 15 a $26^{\circ} \mathrm{C}$ durante a execução do experimento. $O$ plantio foi realizado a 7 de julho de 1980 , com quatro sementes de cada cultivar Forrest o Biloxi por vaso, sendo

(') VALADARES, J. M. A. \& CAMARGO, O. A. Manganês en solos do Estado de São Paulo. (Comunicação pessoal) 
efetuado desbaste posterior para uma planta de cada cultivar por vaso. $\mathrm{Na}$ época da coleta de plantas, foram dadas notas, de acordo com a seguinte escala:

Nota 1; sintomas de necrose e enrugamento a partir das folhas primárias;

Nota 2: sintomas de necrose e enrugamento a partir da primeira folha trifoliada;

Nota 3: sintomas de necrose e enrugamento a partir da segunda folha trifoliada;

Nota 4: sintomas de necrose e enrugamento a partir da terceira folha trifoliada;

Nota 5: ausência de sintomas por ocasião da coleta da parte aérea.

Vinte e sete dias após o plantio, tanto a parte aérea como a radicular foram coletadas, lavadas com água comum e, em seguida, com água destilada, secas a $60^{\circ} \mathrm{C}$ e pesadas. A amostra da parte aérea das plantas, após o seu devido preparo, foi submetida à análise química, segundo a metodologia descrita por BATAGLIA et alii (3).

\section{RESULTADOS E DISCUSSÃO}

As amostras dos solos em estudo mostraram teores elevados de fósforo e potássio, enquanto os teores de $\mathrm{Ca}$ e $\mathrm{Mg}$ disponíveis são baixos, exceto para a amostra de Campinas, conforme é apresentado no quadro 1 . O teor de alumínio na amostra de Cândido Mota é elevado, verificando-se ainda que, à medida que decresce o teor de cálcio, aumenta o de Mn.
As notas do quadro 2 indicam que, no solo de Campinas, os dois cultivares em estudo não mostraram nenhum sintoma de toxicidade de manganês, tendo o solo apresentado 2,9 ppm de Mn solúvel em DTPA. No solo de Guaíra, 'Forrest' apresentou sintomas iniciais de toxicidade de $\mathrm{Mn}$, os quais consistem em enrugamento foliar com necrose da nervura, enquanto em 'Biloxi' não se manifestaram tais sintomas, mesmo sendo o teor de $\mathrm{Mn}$ solúvel em DTPA cerca de 2,2 vezes superior ao de Campinas. Pelos sintomas apresentados, o efeito de toxicidade ao 'Forrest' foi drástico no solo de Guatapará, e mais acentuado ainda em Cândido Mota (Figura 1). Nos solos desses dois municípios, o cultivar Forrest mostrou, além de enrugamento foliar, a necrose da nervura, manchas de coloração escura e nanismo da planta com internódios curtos, enquanto no Biloxi esses sintomas foram mais moderados. Observa-se também que os teores de Mn no solo de Guatapará e de Cândido Mota foram quatro vezes maiores em relação aos do de Campinas.

A análise de variância de peso seco da parte aérea e das raízes mostrou que houve diferenças de comportamento de cultivar independente do solo. A interação cultivar $x$ solo foi significativa a $5 \%$, indicando que os cultivares tiveram comportamento diferente nos quatro solos estudados. Ainda pelo quadro 2, observa-se que, no solo de Campinas, as diferenças são significativas, tanto para peso seco da parte aérea como para o das raízes, entre 'Biloxi' e 'Forrest', sendo essas diferenças inerentes ao fator cultivar. $O$ mesmo 

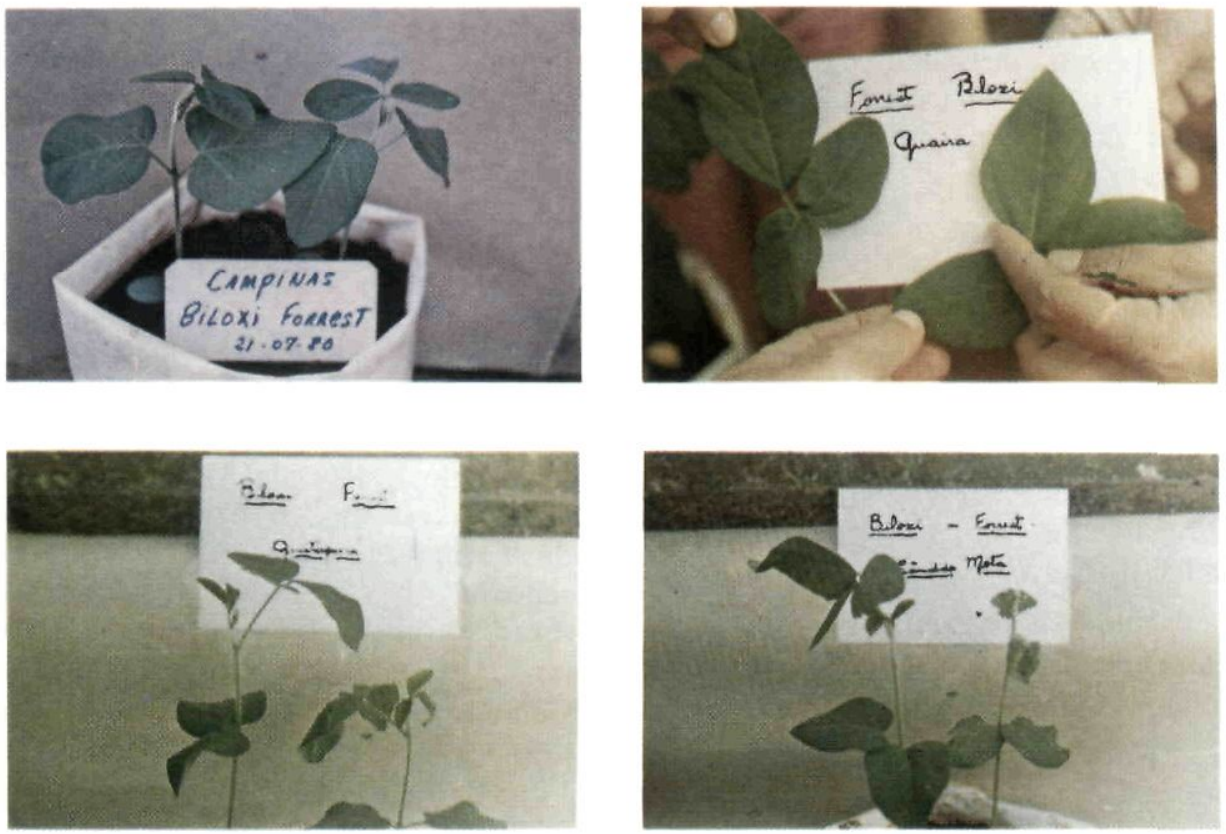

Figura 1. - Comportamento dos cultivares Biloxi e Forrest em solos de Campinas, Guaíra, Guatapará e Cândido Mota

pode ser dito para os cultivares em solo de Guaíra, apesar de o Forrest ter apresentado pouco sintoma de toxicidade de $\mathrm{Mn}$, o qual, todavia, não afetou a produção de matéria seca da parte aérea ou das raízes. Para os solos de Guatapará e Cândido Mota, o cultivar Biloxi apresentou redução de peso da parte aérea da ordem de 1,75 e 1,57 vez, quando comparado com os dados de Campinas, e no 'Forrest' esta redução foi 3,21 e 2,64 vezes respectivamente. Verifica-se certa concordância entre as notas dadas para sintomas visuais de toxicidade de $\mathrm{Mn}$ e quantidade de matéria seca da parte aérea produzida pelos cultivares. A toxicidade de Mn reduziu o peso seco das raízes de Forrest nos solos de Guatapará e Cândido Mota em aproximadamente cinco vezes, quando comparado com o de Campinas. Os altos teores de manganês no solo de Guatapará e Cândido Mota induziram elevados teores deste elemento na parte aérea, conforme pode ser observado no quadro 3 . Os teores determinados são considerados tóxicos para as plantas de soja (19) e o cultivar Forrest mostrou ser bastante suscetível à toxicidade de Mn.

A análise química da parte aérea das plantas dos dois cultivares 


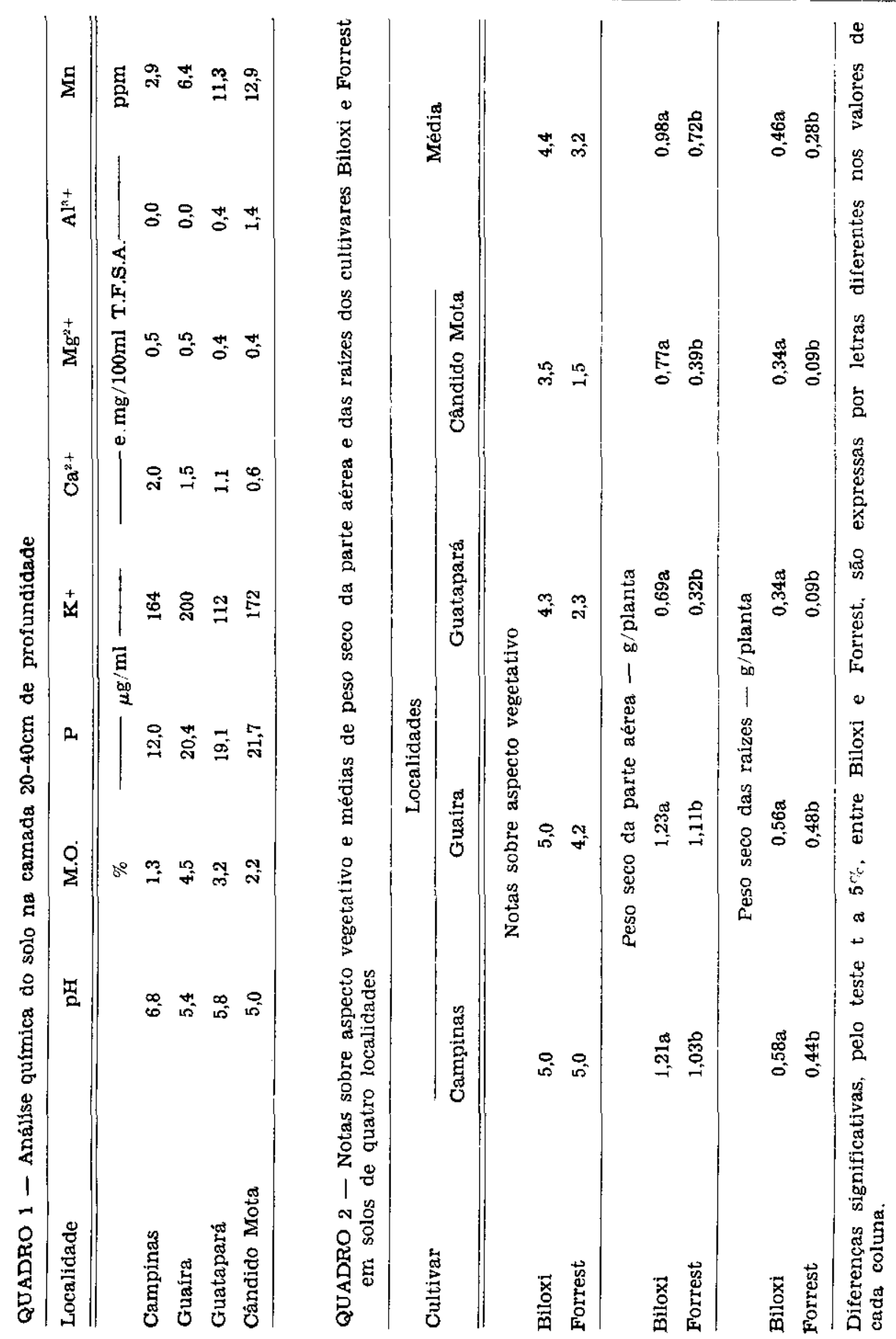




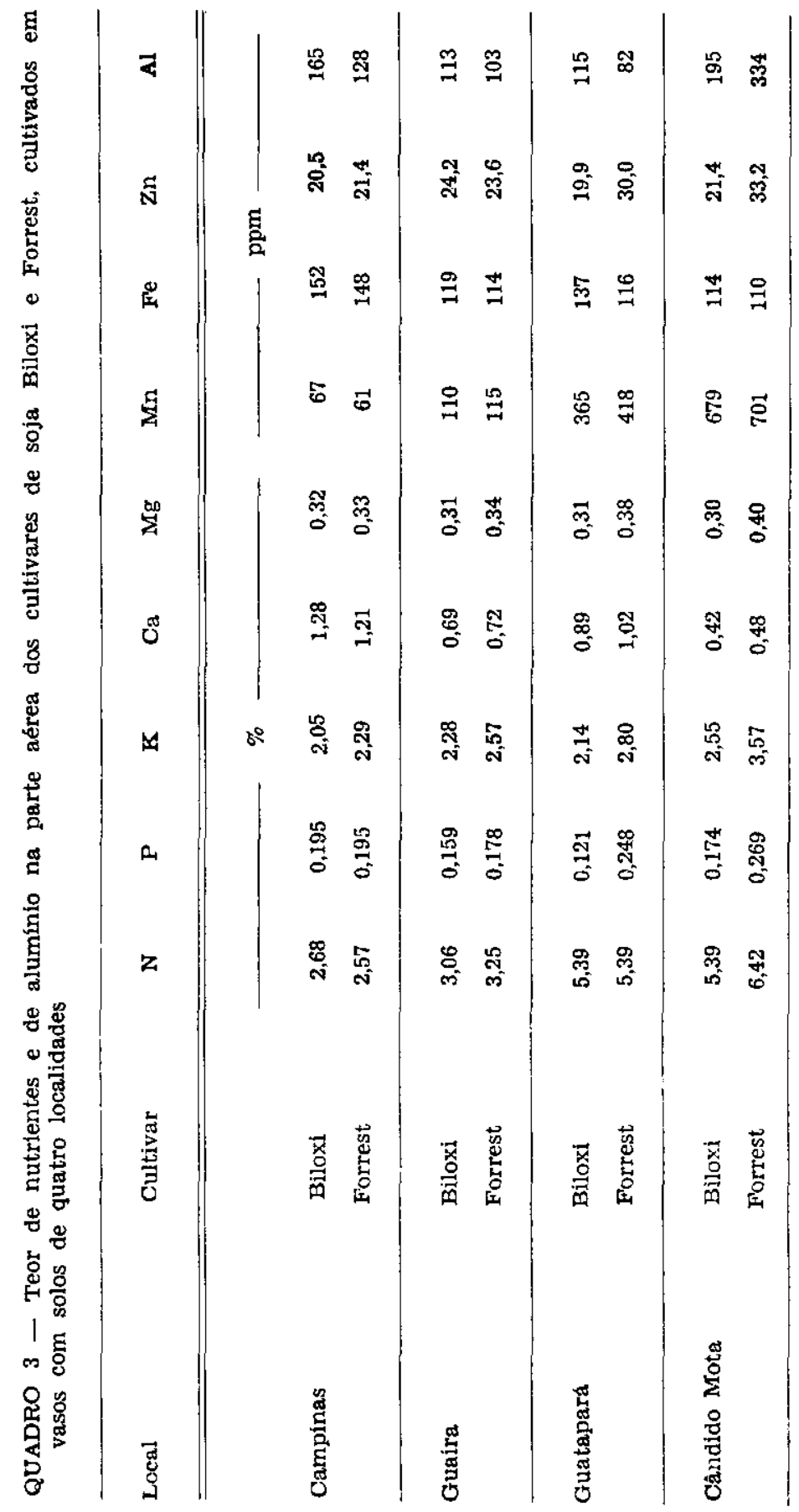


nas quatro localidades (Quadro 3) revelou que houve maior concentração de nitrogênio onde apareceram sintomas de toxicidade de $\mathrm{Mn}$, isto é, em Guatapará e Cândido Mota. Notou-se também maior concentração de fósforo e potássio no cultivar Forrest desenvolvido nos solos destas duas localidades, o qual sofreu ainda os maiores danos com a toxicidade de Mn. Conforme a tabela de Jones, citada por OHLROGGE \& KAMPRATH (20), os teores de Mn em 'Biloxi' e 'Forrest', em solos de Campinas, estão em níveis suficientes; em Guaíra, em níveis elevados; e em Guatapará e Cândido Mota, em níveis excessivos, que causaram, em ambos, os sintomas de toxicidade já mencionados. Observa-se, ainda, que os teores de Mn na parte aérea nos dois cultivares correspondem ao aumento no teor deste elemento, verificado no solo (Quadro 1). Segundo os critérios mencionados (20), os teores de $\mathrm{Ca}, \mathrm{Mg}, \mathrm{Zn}$ e $\mathrm{Fe}$ estão em níveis suficientes para ambos os cultivares nas quatro localidades.

Os dados do quadro 4 referem-se às quantidades de $\mathrm{Mn}$ absorvidas pelas plantas de Biloxi e Forrest e a um índice considerado a partir da relação entre o teor de $\mathrm{Mn}$ da parte aérea e o peso desta (17). Observa-se que, nos solos de Guatapará e Cândido Mota, 'Biloxi' absorveu aproximadamente duas vezes mais Mn que 'Forrest'; entretanto, este último foi mais afetado em seu desenvolvimento pela toxicidade. Comparando-se, todavia, os índices calculados, verifica-se a concordância com os valores das notas e de peso seco apresentados no quadro 2. Essa concordância vem confirmar que a utilização do critério de notas é uma ferramenta importante para o melhorista executar o "screening" de materiais genéticos resistentes à toxicidade de $\mathrm{Mn}$, ao invés de verificar o peso de matéria seca e os teores nas plantas, que são métodos demorados e onerosos.

Conforme relatado por OHKI et alii (19), 'Forrest', em solução nutritiva que continha uma quantidade mínima de $\mathrm{Mn}$, foi o único, dentre os cultivares testados, que não mostrou deficiência de $\mathrm{Mn}$, o que comprova que ele é eficiente em absorvê-

QUADRO 4 - Absorçāo de $\mathrm{Mn}$ e relação entre os teores de $\mathrm{Mn}$ e o peso seco da parte aérea

Cultivar Campinas Guaira Guatapará Cândido Mota

$\mu \mathrm{g} /$ planta

\begin{tabular}{lllll} 
Biloxi & 81 & 135 & 252 & 522 \\
Forrest & 62 & 127 & 133 & 273 \\
\hline
\end{tabular}

Teores de $\mathrm{Mn} /$ peso seco

$\begin{array}{lrrrr}\text { Biloxi } & 56 & 90 & 529 & 882 \\ \text { Forrest } & 60 & 104 & 1.307 & 1.798\end{array}$


-lo. Em Guaíra, foram observados sintomas de toxicidade de $\mathrm{Mn}$, apesar de não terem ocorrido interferências na produção de matéria seca da parte aérea (Figura 1). Como a análise de solo em relação ao tcor de Mn não é um critério útil para leguminosas (12) e a foliar é demo- rada e onerosa, sugere-se a utilização do cultivar Forrest como planta indicadora da toxicidade de Mn no solo (Latossolo Roxo distrófico).

Quanto ao 'Biloxi', os dados obtidos referentes à sua tolerância a elevados níveis de $\mathrm{Mn}$ confirmam os de MASCARENHAS et alii (16).

\section{DIFFERENTIAL MANGANESE TOLERANCE OF TWO SOYBEAN CULTTVARS}

\section{SUMMARY}

A pot study was made in the greenhouse using soybean cultivars Biloxi and Forrest and sub-soil samples of Dusky Red Latosol distrophic soil of four counties namely Campinas, Guaíra, Guatapará and Cândido Mota of the State of Săo Paulo, which contained 2.9,6.4, 11.3 and $12.9 \mathrm{ppm}$ of DTPA pH 7.3 soluable Mn. The Mn toxicity was evalued 27 days after planting for visual symptoms, dry matter production of above ground parts and roots, and chemical analyses of dry matter of above ground parts. The cultivar Biloxi did not show visual symptoms at the $6.4 \mathrm{ppm}$ level of $\mathrm{Mn}$ in the soil (Guaira) whereas Forrest showed initial symptoms of $\mathrm{Mn}$ toxicity at this level. As the $\mathrm{Mn}$ availability in the soil increased there was greater evidence of $\mathrm{Mn}$ toxicity symptoms and less dry matter production. The cultivar Forrest showed to be most affected by $\mathrm{Mn}$ toxicity which was confirmed by increase in the $\mathrm{Mn}$ concentration of dry matter of the above ground parts. As Forrest showed to be more efficient in the uptake of $\mathrm{Mn}$ from the soil and also showed visual symptoms of toxicity at low levels it can be used as an indicator of $\mathrm{Mn}$ in the soil. A good relationship between scores given for visual symptoms, dry matter production for the above ground parts, and the index (Mn concentration/dry matter production) shows the possibility, that scores for visual symptoms of Mn toxicity can be utilized efficiently, for screening genetic material tolerant to $\mathrm{Mn}$ in a soybean breeding program.

\section{REFERENCLAS BIBLIOGRAFICAS}

1. ALMEIDA, A. M. R. \& SFREDO, D. J. Encrespamento foliar e nanismo de plantas de soja associadas à toxidez de manganês. Fitopatologia Brasileira, Brasilia, 4:333-335, 1979 .

2. ANDREW, C. S. \& HEGARTY, M. P. Comparative response of manganese to excess of eight tropical and four temperate legumes. Australian Journal of Agricultural Research, 20:687-696, 1969.

3. BataGlia, O. C.; TEIXEIHA, J. P. F.: FURLANI, P. R.; FURLANI, A. M. C.; GALLO, J. R. Análise química de plantas. Campinas, Instituto Agronômico, 1978. 32p. (Circular, 87)

4. BROWN, J. C. \& JONES, W. E. Fitting plants nutritionally to soils. I. Soybeans. Agronomy Journal, 69:299-404, 1977.

5. CARTER, O. G.; ROSE, I. A.; RFADING, P. F. Variation in susceptibility to manganese toxicity in 30 soybeans genotypes. Crop Science, 15:730-732, 1975.

6. COUTINHO, C.; FREIRE, J. R. J.; VIDOR, C. Comportamento de variedade de soja em relaçăo à toxidez de Al e $\mathrm{Mn}$ de solo ácido do Rio Grande do Sul. Agronomia Sulriograndense. Porto Alegre, 5:133-141, 1971. 
7. FOY, C. D. \& SCHWARTZ, J. W. Opposite aluminum and manganese tolerance of two wheat varieties. Agronomy Journal, 65:123-126, 1973.

8. - FLEMING, A. L.; ARMINGER, W. H. Differential tolerance of cotton varieties to excess manganese. Agronomy Journal, 61:690-694, 1969.

9. FRANCO, A. A. \& DóBEREINER, J. Toxidez de manganês de um solo ácido na simbiose soja-rhizobium. Pesquisa Agropecuária Brasileìra, Brasilia, 6:57-66, 1971.

10. HEENAN, D. P. \& CARTER, O. G. Tolerance of soybean cultivars to manganese toxicity. Crop Science, 16:389-391, 1976.

11. Influence of temperature on the manganese toxicity by two soybean varieties. Plant and Soil, 47:219-227, 1977.

12. HELYAR, R. R. Effects of aluminum and manganese toxicities on legume growth. In: ANDREW, C. S. \& KAMPRATH, E. J. Mineral nutrition of legumes in tropical and sub-tropical soils. Melbourne, Australia, Commonwealth Scientific and Industrial Research Organization, 1978. p.207-231.

13. LINDSAY, W. L. \& NORWELL, W. A. Development of a DTPA micronutrient soil test. Agronomy Abstracts, 1969. p.84.

14. LOHNIS, M. P. Manganese toxicity in field and market garden crops. Plant Soils, 3:193-222, 1951.

15. MASCARENHAS, H. A. A.; BRAGA, N. R.; BUlISANI, E. A.; TEIXEIRA, C. T.; HIROCE, R.; BATAGLIA, O. C. Efeito do corretivo sobre soja cultivada em solo de cerrado contendo Al e Mn. In: SEMINARIO NACIONAL DE PESQUISA DE SOJA, 2., Brasilia, DF, 1981. Resumos. p.171.

16. —; MIRANDA, M. A. C.; RAMOS, L. C. S.; FURLANI, P. R.; BATAGLIA, O. C. Comportamento diferencial de três cultivares de soja em diversos níveis de manganês. In: REUNIAO BRASILEIRA DE FERTILTOADE DO SOLO, 15., Campinas, 1982. Resumos. p.22. (Resumo 60)

17. MORAES, F. R. P. de; GALLO, J. R.; IGUE, T.; FIGUEIREDO, J. I. Efeito de três fertilizantes acidificantes sobre a concentraçăo de alumínio e de manganês em folhas e raizes de cafeeiros. Bragantia, Campinas, 38:7-17, 1979.

18. MORRIS, H. D. \& PIERRE, W. H. Minimum concentration of manganese necessary for injury to various legumes in culture solution. Agronomy Journal, 41:107-113, 1949.

19. OHKI, K.; WIISON, D. O.; ANDERSON, O. E. Manganese deficiency and toxicity sensitivities of soybeans cultivars. Agronomy Journal, 72:713-716, 1980.

20. OHLROGGE, A. J. \& KAMPRATH, E. S. Fertilizer use in soybeans. In: DINAUER, R. C., ed. Changing patterns in fertilizer use. Madison, Wisconsin, U.S.A., Soil Sciense Society of America, 1968. p.288.

21. QUELLETTE, G. J. \& DESSUAREAUX, L. Chemical composition of alfafa as related to degree of tolerance of manganese and aluminum. Canadian Journal of Soil Science, 38:206-215, 1958.

22. RAIJ, B. van \& ZULIO, M. A. T. Métodos de análise de solo. Campinas, Instituto Agronômico, 1977. 16p. (Circular, 63) 